\title{
NARRATIVAS DE AGENTES INTERVENTORES DE ABUSO SEXUAL INFANTIL
}

\section{NARRATIVES OF CHILD SEXUAL ABUSE INTERVENTION OFFICERS}

\section{NARRATIVAS DE AGENTES DE INTERVENÇÃO EM ABUSO SEXUAL INFANTIL}

Marcela Velásquez Herrera Universidad Nacional Abierta y a Distancia UNAD marcela.velasquez@unad.edu.co Acacias - Meta

Laura Nathalia González Barragán Universidad Santo Tomás Sede Villavicencio

lauragonzalezb@usantotomas.edu.co Villavicencio - Meta

Henry Farley Gutiérrez Herrán Universidad Santo Tomás Sede Villavicencio henrygutierrez@usantotomas.edu.co Villavicencio - Meta

Neidy Mabelyn León Roldán Universidad Santo Tomás Sede Villavicencio neidy.leon@usantotomas.edu.co Villavicencio - Meta

Diagramación e llustración de Portada Sindy Catherine Charcas Ibarra

Encuentre este artículo en:

http://revistas.uniminuto.edu/index.php/IYD

Para citar este artículo / To cite this article

Velásquez -Herrera, M; González Barragán, L; Gutierrez-Herrán, H; León-Roldán, N. (2020). Narrativas de agentes interventores de abuso sexual infantil. Inclusión \& Desarrollo, 7 (2), pp. 37-49
Fecha de recepción: 20 de febrero de 2020

Fecha de aceptación: 17 de mayo de 2020

Fecha de publicación: 30 de junio de 2020 


\section{RESUMEN}

El presente artículo busca presentar las reflexiones derivadas del estudio "Narrativas de agentes interventores en torno a la atención del abuso sexual infantil", el cual tuvo como objetivo comprender las narrativas que se configuran en agentes interventores en torno a la atención de casos Abuso Sexual Infantil. Para ello se elaboraron escenarios conversacionales, enmarcados en el método de hermenéutico interpretativo, con el fin de favorecer la construcción de manera conjunta de narrativas a partir de la deconstrucción de los relatos dominantes y saturados expresados por los actores de la investigación, quienes fueron tres agentes interventores con experiencia en la atención de casos de abuso sexual infantil. Los resultados visibilizan diversas narrativas en torno a su proceso autorreferencial, el cual está inmerso en su rol como agente interventor, viéndose de esta manera afectado por experiencias personales y laborales. Asimismo, se establecen narrativas de satisfacción con su labor, ya que cuentan con recursos internos y externos, como los lineamientos establecidos por la institución, recursos de autoorganización, como la llamada "memoria" y el trabajo interdisciplinar, que resalta en la labor de intervención de casos de abuso sexual infantil. Por otro lado, se manifestaron posturas sobre el rol de la familia, la cual, si bien en ocasiones puede ser dominante y problemática, también se le reconoce su capacidad de cambio y la importancia de esta para el trabajo en la prevención.

Palabras clave: agentes interventores, narrativas, abuso sexual infantil, autorreferencia.

\section{ABSTRACT}

This article seeks to present the reflections derived from the study "Narratives of intervening agents around the attention of child sexual abuse", which aimed to understand the narratives that are configured as intervening agents regarding the attention of cases of Child Sexual Abuse. For this, conversational scenarios were elaborated, framed in the interpretative hermeneutic method, in order to favor the joint construction of narratives from the deconstruction of the dominant and saturated stories expressed by the research actors, which were three intervention agents with experience in the care of cases of child sexual abuse. The results make visible various narratives around his self-referential process, which is immersed in his role as an intervention agent, thus being affected by personal and work experiences. Likewise, narratives of satisfaction with their work are established, since they have internal and external resources, such as the guidelines established by the institution, self-organization resources such as the so-called "memory" and interdisciplinary work which is highlighted in the intervention work. of child sexual abuse cases. On the other hand, positions were expressed on the role of the family, which, although it can sometimes be dominant and problematic, is also recognized for its capacity for change and its importance for work in prevention.

Keywords: intervention agents, narratives, child sexual abuse, self-reference.

\section{SUMÁRIO}

Este artigo busca apresentar as reflexões derivadas do estudo "Narrativas de agentes intervenientes em torno da atenção ao abuso sexual infantil", que objetivou compreender as narrativas configuradas nos agentes intervenientes em relação à atenção aos casos de abuso sexual infantil . Para isso, foram elaborados cenários conversacionais, enquadrados no método hermenêutico interpretativo, a fim de favorecer a construção conjunta de narrativas a partir da desconstrução das histórias dominantes e saturadas expressas pelos atores da pesquisa, que eram três agentes Intervenientes com experiência no 
atendimento de casos de abuso sexual infantil. Os resultados tornam visíveis várias narrativas em torno de seu processo autorreferencial, imerso em seu papel de agente de intervenção, sendo afetado por experiências pessoais e profissionais. Da mesma forma, são estabelecidas narrativas de satisfação com o trabalho, pois possuem recursos internos e externos, como as diretrizes estabelecidas pela instituição, recursos de auto-organização, como a chamada "memória" e o trabalho interdisciplinar, que se destacam no trabalho de intervenção. de casos de abuso sexual infantil. Por outro lado, foram expressas posições sobre o papel da família, que, embora às vezes possa ser dominante e problemático, também é reconhecida por sua capacidade de mudança e sua importância no trabalho de prevenção.

Palavras chave: agentes de intervenção, narrativas, abuso sexual infantil, auto-referência. 


\section{Introducción}

El abuso sexual infantil (ASI) es un fenómeno social de gran magnitud en el mundo. En Colombia las cifras revelan que, de 25.695 denuncias registradas de delitos sexuales en el año 2019, el 86,5\% son casos de abuso sexual infantil (Instituto de Medicina Legal y Ciencias Forenses ,2019). Considerando estas cifras, muchos autores han generado comprensiones en torno al fenómeno de abuso sexual infantil, centrándose en diferentes focos, como narrativas del suceso, causas, consecuencias e intervención entre otros. Asimismo, se observan investigaciones que resalta los tipos de terapias que se han abordado en niños, niñas y adolescentes (NNA) víctimas de abuso sexual, haciendo relevante la atención en procesos individuales y de los sistemas sociales involucrados desde la formulación de acciones que favorecen el proceso de reparación, reconstrucción de significados y estrategias de afrontamiento (Bustamante, 2016; Rodríguez, 2015; Capellán y Gutiérrez, 2014).

Lo anterior, denota un gran interés investigativo alrededor de quienes vivencian directamente un acontecimiento de abuso, invisibilizando las voces de quienes brindan una atención a esta problemática social, por ello es importante entender que en el fenómeno del ASI, se teje un entramado de relaciones constituido por las familias, las instituciones y la comunidad en general, los cuales se convierten en actores activos y que requieren de una labor en red para sus intervenciones (Águila et al., 2017; Sellado, 2015; Barreto, 2013). Por otro lado, estudios alternos resaltan el funcionamiento familiar, como un factor de vulnerabilidad o protector para el niño, niña o adolescente; dando importancia a cómo se estructura y reestructura este sistema a raíz de la vivencia del ASI (Villanueva et al., 2011; Quiroz Peñaranda, 2009). De igual manera, diversos autores resaltan las voces de las familias ya que estas cumplen un importante papel en la problemática social del ASI, configurando su visión del fenómeno, sus causas, el perfil del abusador y su prevalencia; generando cambios significativos en las comprensiones de la víctima sobre lo vivido (Pereda y Sicilia, 2017; Betancourt, 2016; Gaviria, 2015).

El presente artículo da a conocer el resultado obtenido del proceso investigativo titulado, Narrativas de agentes interventores en torno a la atención del abuso sexual infantil en la ciudad de Villavicencio, desarrollada en el 2019, donde se buscó resaltar las voces de los agentes interventores como actores activos en el entramado relacional que trae consigo el ASI, estos reconocieron la importancia de su rol, sus procesos autorreferenciales, la institucionalidad y la importancia del trabajo interdisciplinar para intervenir en el fenómeno del ASI.

El ejercicio investigativo propuesto se enmarcó desde el pensamiento complejo- socio construccionista- constructivista, el cual invitó a que emergieran nuevos significados en los actores, en la práctica de lenguajear, y en la comprensión de la construcción de narrativas de los participantes. Para ello se tuvo en cuenta, el marco referencial de las narrativas, los roles del agente interventor y el ASI.

\section{Marco teórico}

\section{Narrativas}

En el campo académico las narrativas se utilizan para comprender las historias, al realizar un análisis que atiende a la estructura, la temporalidad, la temática y a los significados que están a un proceso continuo de interpretación subjetiva. Todos estos elementos construyen los relatos que denominamos narrativas y que Domínguez y Herrera (2013) refieren como "Ios instrumentos primordiales para la creación y comunicación del significado." (p.5).

Por otra parte, para la investigación las narrativas han sido comprendidas desde dos perspectivas: la construccionista social y el 
constructivismo (Domínguez y Herrera, 2013). La mirada construccionista social, comprende la narrativa como parte de la construcción hecha no por sujetos a nivel individual, sino desde la interacción donde se construyen mundos, significados y realidades conjuntas, por lo cual no pueden aislarse de los atributos propios de la interacción social como lo son los referentes y los marcos de interpretación que estarán en relación con una determinada audiencia, en un momento y contexto particular; la segunda perspectiva se desarrolla desde el constructivismo y hace énfasis en la función de las narrativas como medio para construir significados de las experiencias y del mundo en el que viven los sujetos, de esta forma los significados son las representaciones del mundo y lo que en él hay. Asimismo, se señala como otra característica de la narrativa desde esta perspectiva su origen vinculado a la condición humana del ser como un sujeto socialmente interdependiente, por lo que se reconoce que se generan a partir de la interacción, pero a su vez cada narrativa es única e individual en tanto las motivaciones, emociones y cogniciones serán particulares en cada sujeto.

Se rescata la propuesta de Payne (2002), sobre las narrativas quien las define como "las secuencias escogidas de vida que cobran existencia como entidad mediante el acto de relatarlas" (p. 36) de esta forma el narrar no solo da una descripción y reflejo de la vida de los sujetos, sino que la consolida, entendiéndose de esta forma como una oportunidad favorecedora de cambio, puesto que todo cambio sucede de la narración y, por ende, toda aquella intervención cambia la narración que realiza de sí misma.

El estudio sobre el que se basa el presente artículo resalta la existencia de narrativas dominantes y alternas, dos tipos de narrativas que fueron reconocidas y comprendidas inicialmente desde el ámbito terapéutico al referirse a las narrativas como eje central de la terapia, pues se afirma que los seres humanos organizan su propia experiencia de vida en forma de una narración
(Payne, 2002). En este sentido, López (2011) expresa que las narrativas dominantes son aquellas que restringen nuestro ser, que excluyen aspectos relevantes de la experiencia y/o llevan al sujeto a conclusiones negativas sobre su propia identidad, estos relatos imperan en la vida, marcando el curso del comportamiento de las personas y saturando la existencia de la problemática en que se encuentra.

De la misma manera, López (2011) se refiere a las narrativas alternas como aquellas que difieren a las narrativas dominantes y saturadas del problema, ya que buscancentrar las comprensiones de los problemas, de los significados y de la vida misma desde otras experiencias que no han sido visibilizadas por lo que es necesario nutrir este tipo de relatos para que cobren un papel primordial en la vida del sujeto, posibilitando cambios y transformaciones de su realidad, hecho que favorece el bienestar psicológico de los sujetos. Por otra parte, se resalta en los contextos narrativos la emergencia de la autorreferencia que Celis y Rodríguez (2016) describen como la posibilidad de consciencia de lo individual desde lo relacional, reconociéndose como un proceso constructivista que refiere un observar hacia sí mismo a partir del otro.

De forma concreta, Garzón (2008) remite la noción de autorreferencia como un "proceso recursivo de observar las observaciones; de este modo se da cuenta de la reflexividad en contexto para adquirir conciencia de sí mismo, del otro y del nosotros que emerge en los encuentros humanos" (pp.161-162). Por ello abordar la autorreferencia posibilita construir comprensiones más amplias de las intervenciones realizadas por los profesionales y de la misma forma da cuenta de la condición humana de estos interventores, hecho que se dirige a una apuesta ética ya que propende por mayor responsabilidad social y ética en las intervenciones.

\section{Agentes interventores}

El Instituto Colombiano de Bienestar Familiar (2017), En adelante (ICBF, 2017); define 
que un agente de intervención es entendido como una persona cualificada que ejerce como instrumento para realizar intervenciones con familias o con individuos, por ello son profesionales de diversas áreas (abogados, psicólogos, nutricionistas, trabajadores sociales, etc) que atienden problemáticas sociales cuestionando sobre la forma de ver a las familias y simultáneamente desarrollan un trabajo en las competencias para que se generen procesos transformadores en las vivencias familiares que establecen los motivos de intervención. En cortas palabras, el agente interventor se convierte en un puente entre la familia, su entorno, la institución y el niño.

El agente interventor debe trabajar bajo la ética compleja y sistémica que sustentarán las acciones que genere con los consultantes, esto a independencia de su profesión o rol al interior de la institución (ICBF, 2017). Por ello sus actuaciones dan importancia a los vínculos e interacciones entre los individuos de un determinado sistema.

Asimismo, se reconoce que cada sistema mantiene unos objetivos, todos los miembros del sistema nuevo que se genera entre agentes interventores y familias, establecen unas finalidades, cada miembro tiene un objetivo distinto que estará relacionado a los conocimientos generados en torno al problema. Los agentes interventores de distintas disciplinas son actores activos con autonomía y poder para establecer estrategias de intervención acordes con los conocimientos que definen su quehacer profesional. Por lo anterior cada agente, en el marco de un equipo profesional y de una institución, debe evaluar los objetivos a alcanzar y de qué manera lo lograrán, hecho que determinará la forma cómo se relacionen los agentes interventores (ICBF, 2017).

Finalmente, los agentes interventores deben esforzarse por comprender la realidad que exponen los consultantes, entendiendo que no existen soluciones únicas, pues cada familia cuenta con recursos diferentes y el profesional solo realiza un acompañamiento de ese proceso de cambio que genera la familia. Sin embargo, es inevitable que los agentes interventores empiecen a crear preconceptos de las problemáticas a intervenir en las familias, hecho que se suma al seguimiento de políticas y lineamientos establecidos para la intervención, donde la atención al usuario llega a ser insuficiente, situaciones que favorecen la creación de prejuicios acerca de los sistemas atendidos, lo cual terminará en la ralentización de los procesos, generando sentimientos de culpa y juicios en las familias, lo que imposibilita que se empoderen de aquellos recursos con los que cuentan para transformarse y construir nuevas realidades, superando la situación problemática como lo es un acontecimiento de ASI (ICBF, 2017).

\section{Abuso Sexual Infantil (ASI)}

Una definición del Abuso Sexual Infantil presentada por Kamsler (1990) establece que es:

un acto sexual infligido a una persona joven o a un niñoporotrapersona.Lacapacidaddecomprometer a un niño en una relación sexual se base en la posición todopoderosa y dominante del adulto (o de un adolescente mayor que el niño agredido) ultrajador, posición que contrasta agudamente con la edad, la dependencia y la impotencia del niño. La autoridad y el poder permiten que el agresor obligue al niño a someterse sexualmente (p.16).

Por otra parte, Corsi (1994, citado en Rúa, Pérez y González, 2018) señalan que este tipo de abuso puede ser intrafamiliar o extrafamiliar y según los registros de violencia sexual contra niños, niñas y adolescentes el abuso sexual se produce mayormente en el interior de las familias.

De la misma forma Rúa, Pérez y González (2018) refieren diversas categorías del ASI: abuso sexual, agresión sexual, exhibicionismo y la explotación sexual, adicionalmente, en la actualidad se reconocen nuevas formas como el abuso sexual entre iguales y el grooming que son los realizados 
por medio de la virtualidad como la obtención de imágenes o videos sexualizados de la persona menor llegando hasta la ejecución del abuso. Por lo anterior, el concepto de ASI se ha transformado y no solo se reduce a las interacciones abusivas de una persona adulta a una menor.

Por lo anterior, se comprende que las consecuencias que conlleva un acontecimiento de abuso son múltiples físicas, cognitivas, comportamentales emocionales y sociales, varían en cada persona, por eso no es posible hablar de un síndrome del abuso sexual (Pereda y Sicilia, 2017)

\section{Metodología}

Planteada la importancia y densidad del fenómeno de investigación, se llevó a cabo el ejercicio investigativo desde la metodología cualitativa. De esta manera, se pudo comprender e interpretar, aquellos relatos, discursos, narrativas de los participantes sean los agentes interventores o los investigadores. Esto último, para favorecer la construcción de manera conjunta de narrativas a partir de la deconstrucción de los relatos dominantes y saturados visibilizados desde la estrategia de escenarios conversacionales. Por ello, es claro que la idea de una narrativa única al conversar sobre un fenómeno, no existe, y que aquellas que emerjan son iguales de verdaderas y válidas según Quecedo y castaño (2002).

Para lograr lo mencionado, el método de la hermenéutica interpretativa fue fundamental al proceso investigativo, puestoque,estasepreocupa por interpretar y comprender el comportamiento humano, además comprende el texto y el discurso desde sus significados, emoción, subjetividad, y las intenciones de quien lo interpreta (Anderson, 2012). Por ello al hacer uso de esta metodología, el objetivo estuvo direccionado a comprender los significados construidos en torno al ASI desde la postura autorreferencial, de los agentes interventores, producto de su experiencia profesional.
Como se ha mencionado anteriormente, los actores partícipes y protagonistas de este entramado investigativo, fueron tres agentes interventores, profesionales desde las disciplinas de derecho, psicología, y trabajo social que laboraban en un Centro de Atención a Víctimas de Abuso Sexual. Se estableció, como principal criterio de inclusión que estas personas tuvieran experiencia en la atención e intervención de casos de ASI. En virtud, de la ética profesional del trabajo investigativo, se establece un consentimiento informado, donde se atiende al principio de confidencialidad por lo que se presentan seudónimos para cada participante.

En coherencia con los principios metodológicos, la estrategia escogida para la obtención de las narrativas fueron los escenarios conversacionales propuestos por Estupiñán, González y Serna (2006, citados en Aya, 2012) dada la facilidad que genera para la evocación de historias, narrativas vivenciales y experienciales de los participantes. Además, de posibilitar resignificaciones de conceptos, para una transformación de acción e interrelación con el fenómeno desde los actores y los investigadores. Por otro lado, cabe aclarar, que el uso de estos escenarios en la propuesta investigativa fue de carácter académico sin alguna intención interventiva en los participantes, sino como estrategia para favorecer procesos autorreferenciales.

Cada escena de dicha estrategia se desarrolló desde unos focos y objetivos teniendo en cuenta el interés de la investigación y los objetivos que como estrategia presentan los escenarios conversacionales, pues según Estupiñán y González (2015) estos son:

Primero, Evocar y explorar reflexivamente las narrativas dominantes, los contextos relacionales/ conversacionales y las pautas relacionales que los organizan, donde emergen tales narrativas y que estas a su vez, ayudan a mantener. Segundo, Comprender y deconstruir sus significados 
vividos y sus efectos pragmático en la vida de los dilemas. Tercero, Co-construir narrativas alternas que negocien y coordinen nuevos significados posibilitadores de nuevas experiencias, acciones y pautas de relación (p. 96).

Siendo así, la primera escena buscó evocary explorar las narrativas dominantes, y comprender las pautas de las dinámicas de interrelación al momento de la atención, es decir desde las voces de los agentes interventores. La segunda escena planteó comprender y deconstruir los significados vivenciados en las narrativas emergentes de la primera escena, desde las voces de los investigadores. Finalmente, la tercera y cuarta escena, se direccionó a la co construcción de narrativas alternas que conciliarán y coordinarán nuevos significados favorecedores de nuevas acciones y pautas de atención e interacción en ASI.

Posteriormente a la aplicación de la estrategia de investigación, se lleva a cabo la debida sistematización de la información, e igualmente el registro de audio, los consentimientos informados, las matrices de interpretación y finalmente la codificación de los participantes.

De esta forma se comprende el procedimiento de los escenarios conversacionales como la exploración de narrativas de atención de ASI, a partir de los cuales se busca la deconstrucción de relatos dominantes, para construir narrativas colaborativas y finalmente comprender las narrativas alternas y emergentes de los agentes interventores en todo el proceso.

Para brindar mayor claridad del desarrollo de cada escena del escenario conversacional, se presentan algunas figuras que permiten comprender los niveles de observación y escucha, quienes conversan e interactúan en cada momento, la distinción entre participante e investigador, y finalmente los focos y los objetivos de cada escena.

A continuación, se presenta la primera escena llamada "El espejo negro", pretendió evocar y comprender los significados, narrativas dominantes y alternas en torno a la atención del ASI por parte de los agentes interventores. A partir de la siguiente imagen, se comprende que, dos investigadores observan y analizan la interacción conversacional de los participantes, quienes dialogan alrededor de las preguntas orientadoras con uno de los investigadores.

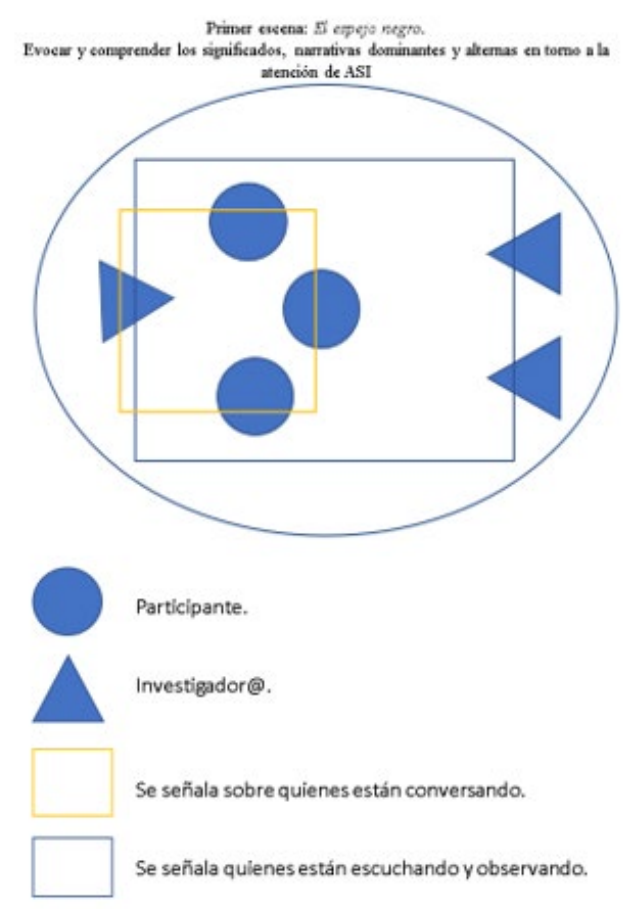

La segunda escena, "El espejo del otro", tuvo como objetivo la deconstrucción de las narrativas dominantes, visibilizar los procesos autorreferenciales de los investigadores y de las participantes, para finalmente reconocer las narrativas emergentes. Por ello, son los participantes quienes observan y analizan lo comprendido por parte de los investigadores acerca de los discursos de la primera escena. En este momento los investigadores empezaron la búsqueda de las posibles narrativas emergentes.

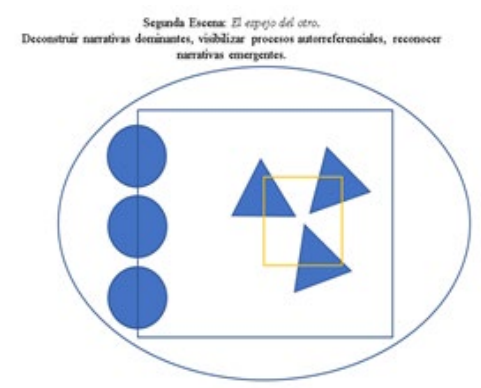


La tercera escena llamada "Reflejo de claridad", tuvo la pretensión de co construir narrativas entre los participantes y los investigadores en torno a la atención del ASI, y de esta manera generar conocimiento colaborativo, visibilizando los procesos autorreferenciales y la emergencia de una nueva categoría de investigación como lo fue, la" institucional", (con el fin de entender los procedimientos y atención por parte de la institución para los interventores y para aquellos a quienes ellos intervienen).

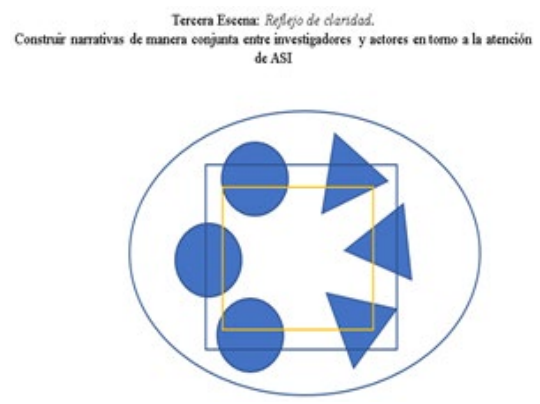

Finalmente, la cuarta escenallamada "El espejo", dio cuenta de las comprensiones finales realizada por parte de los participantes sobre lo conversado en el espacio, dejando de esta manera, la oportunidad de reflexiones propositivas a nuevos cambios.

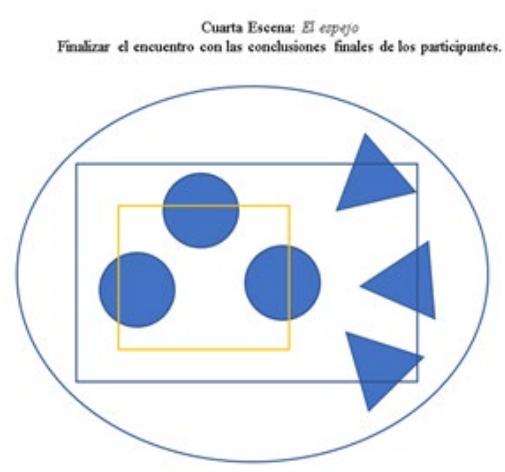

\section{Resultados}

La postura del agente interventor emerge a partir de la comprensión de su autorreferencia, su rol y el proceso de atención. Con ello se entiende que la autorreferencia de los agentes interventores está dada en la construcción de su experiencia laboral, y académica; reconociendo una postura que resalta la perspectiva de justicia y ética profesional, desde la legitimación de un estatus de jerarquía que se muestra fuerte emocionalmente y que evita ser percibido como una persona inestable ante la situación del usuario.

Lo anterior, se entreteje con narrativas alternas de la construcción afectiva de los agentes interventores, transformando el ideal de profesionales justos y neutrales en la atención, hacia el reconocimiento de los dificultades o limitaciones que pueden surgir en el ejercicio laboral cotidiano, de manera que, se vinculan los distintos niveles de atención interdisciplinar o disciplinar con los que se encuentran inmersos los agentes interventores, permitiendo el pensarse los límites de la práctica profesional en la atención.

Sumado a lo anterior, se comprende que el sistema logra identificar sus recursos, como: la buena comunicación y confianza con la que se relacionan sus miembros, con el fin de autoorganizarse, asimilando que esta cohesión que logra el sistema se relaciona con la atención que brinda a los niños y sus familias. De igual forma, cada profesional cumple con una función establecida y el rol que ejercen, sin embargo, en ocasiones se ve el elemento de la borrosidad, planteado desde el pensamiento complejo, al realizar tareas que no estaban establecidas en sus profesiones (Pastor y León, 2007).

En la atención de abuso sexual infantil surge el papel que ejerce el nutricionista. Entendiéndolo como dicha profesión que trabaja con las afectaciones físicas que pueda presentar el niño, niña y adolescente; asimismo, se le da la importancia al trabajo interdisciplinar que realizan los agentes interventores en casos de ASI (Puerto, 2015; Rodríguez, 2003).

El sistema de la defensoría de familia se regeneraconstantemente,elequipointerdisciplinar va cambiando, pero siempre quedará una persona, la cual se denomina "memoria", quien ayudará a continuar con su funcionamiento, esta herramienta moviliza al sistema, para favorecer la adaptación de 
otros agentes interventores que se vinculan, de esta forma se reconoce en este hecho procesos de autoorganización en el trabajo interdisciplinar (Pastor y León, 2007).

Se rescata también la importancia que brinda el marco del proceso de restablecimiento de derechos (ICBF, 2018) en la atención integral a víctimas de abuso sexual infantil, este procedimiento es descrito por los agentes interventores como monótono y desgastante, lo cual limita las posibilidades de evaluar y reconocer las particularidades de cada caso, sustentándose en que la postura del agente interventor ya se encuentra preestablecida y así las acciones que desde el rol se desprenden, por lo que señala una postura distante y rígida en el cumplimiento de la norma.

Por su parte, sobre el ASI se visibiliza el imaginario social y cultural del Abuso Sexual Infantil como el acto coital. Lo cual, expone cómo se presenta la naturalización de esta forma de violencia hacia la infancia, un hecho que se vincula con el desconocimiento que socialmente se presenta respecto a lo que está tipificado como Abuso Sexual y a las afectaciones que se construyen en torno a este fenómeno. Asimismo, desde las narrativas expresadas se configuran no solo en afectaciones individuales de aquel que es reconocido como "víctima", sino de todo el entramado relacional que llega a interactuar alrededor de esta problemática.

Continuamente los agentes interventores resaltan la importancia de la prevención del ASI a nivel familiar y social; describen tipologías familiares específicas y patrones comportamentales que visibilizan disfuncionalidad; se comprende a la familia como favorecedora para la ocurrencia de casos de ASI.

De igual forma se construye la comprensión de la familia que enfrenta una situación de ASI como un sistema que rompe sus dinámicas vinculares, particularmente con la figura materna, dado el rol de cuidado que social y culturalmente ha asumido y que ante un abuso queda invisibilizado, finalmente se reconocen familias que empiezan a co-depender de la atención y que se ven envueltas por este fenómeno, lo anterior ha sido señalado por diversos autores como Velázquez, Delgadillo y González (2013); Villanueva et al. (2011); Quiroz y Peñaranda (2009); Sinclair y Martínez (2006).

En contraste con lo anterior, en la conversación sostenida con los agentes interventores se reconocen narrativas sobre la capacidad de cambio y transformación que tienen las familias, y se plantean procesos en los que la atención favorezca ajustes en la familia como unidad de supervivencia para la generación de ambientes favorables para el bienestar del niño, niña o adolescente (Barreto,2013), señalando así, que es posible en la medida que se tengan mayor tiempo y apoyo interdisciplinar para llevar a cabo los procesos.

\section{Conclusiones}

Se resalta que, para los agentes interventores, su atención es considerada como justa y oportuna enmarcada en el cumplimiento de las rutas de atención y actuaciones orientadas por los lineamientos generados en la institucionalidad. Por otro lado, este mismo camino marcado y determinado puede generar que la atención se torne monótona y llena de preconcepciones sobre las familias, niños, niñas y adolescentes que Ilegan a ser usuarios, en contraposición a esto surgen las narrativas alternas que reconocen la necesidad de una atención que dé cuenta la particularidad de cada sistema que llega a ser intervenido.

Asimismo, atendiendo al objetivo de visibilizar la postura autorreferencial en las narrativas de los agentes interventores, se describió que el rol está permeado por afectaciones de su ejercicio laboral en el desarrollo de la vida personal y otras áreas de desarrollo humano, sin embargo, se rescatan sentimientos de gratificación que fortalece la compresión de un rol como agentes transformadores de cambio. 
Igualmente, en relación con el objetivo de reconocer las narrativas que construyen los agentes interventores alrededor del ASI, continúa siendo dominante la narración social del fenómeno como el acto coital, por lo que permanece el desconocimiento de lo que se enmarca legalmente como ASI, en este sentido, continúa visibilizando la ocurrencia del fenómeno a nivel intrafamiliar. Visto de otra manera, se resalta de forma alterna la importancia de la cultura de la prevención a nivel familiar y social, así como la cultura de la denuncia para contribuir al bienestar de la infancia y la sociedad.

De igual manera, la familia en medio del abuso es comprendida de forma dominante como problemática y disfuncional, en este sentido, el niño al cuidado de las familias llega a verse como sujeto de vulneración constante, siendo así, se reconocen sistemas familiares que pueden verse envueltos por el fenómeno del ASI y dependientes a las intervenciones dadas en la institución, sin embargo, también se reconocen narrativas alternas que resaltan las capacidades de cambio y transformación que poseen las familias y que se dan en sus dinámicas relacionales favoreciendo la resignificación y afrontamiento del evento de abuso en los niños y la familia.

Finalmente, se reconocen otras narrativas dominantes que visibilizan la institución donde se lleva a cabo la atención en casos de ASI, de esta forma la institucionalidad plantea lineamientos determinados en el marco de la atención de dichos casos, sin embargo, se reconoce las falencias en torno a la relación con otras instituciones que intervienen en el fenómeno. También, se reconoce la interdisciplinariedad como un elemento necesario para la atención justa y adecuada dada por la institución, además, se exaltan elementos favorecedores como la buena comunicación, confianza y trabajo en equipo. Al mismo tiempo se reconoce de manera alterna la Institución como organización compleja que se autoorganiza, en este sentido, se resaltan los procesos de rotación de funcionarios, lo cual para las participantes puede comprenderse de forma ambivalente, señalando la necesidad de la permanencia de uno de los miembros del equipo interdisciplinar en cada defensoría como la "memoria" que posibilitará una atención adecuada. 


\section{Referencias bibliográficas}

Águila, D., Beiza, G., Capellán, C., Dussert, D., Gutiérrez, C., Lama, X.y Rodríguez, L. (2017). Narrativas de Padres de Niños, Niñas y Adolescentes que Han Finalizado Psicoterapia por Agresiones Sexuales: Un Proceso de Superación Conjunta. Psykhe, 26(1), 1-14. Recuperado de: https://scielo.conicyt.cl/pdf/ psykhe/v26n1/art04.pdf Anderson, H. (2012). Conversación, lenguaje y posibilidades. Un enfoque posmoderno de la terapia. Buenos Aires, Argentina: Amorrortu.

Aya, S. (2012). Una propuesta de tipo investigativo - interventivo para construir resiliencia. Diversitas: Perspectivas en Psicología, 8(2), 391-406. Recuperado de http://www.redalyc.org/ pdf/679/67925837013.pdf

Barreto, J. (2013). El sistema psico-socio-jurídico como Posibilitador de cambio de narrativas en Familias e instituciones con abuso sexual. Revista Principia luris, 20 (20), 131-150. Recuperado de: http://revistas.ustatunja.edu.co/index.php/piuris/article/view/805/783

Betancourt, I. (2016). Narrativas sobre la violencia sexual en el marco del conflicto armado colombiano. Derecho y Ciencias Sociales, 14(14), 76-103. Recuperado de https://revistas.unlp.edu.ar/dcs/issue/view 1249

Bustamante, M. (2016). Prácticas Narrativas en Psicoterapia con Niños, Niñas y Adolescentes que han sido abusados sexualmente (tesis de pregrado). Universidad de Chile. Santiago, Chile.

Capellán, C. y Gutiérrez, C. (2014). Psicoterapia con niños/as y adolescentes que han sido víctimas de agresiones sexuales: Sobre la reparación, la resignificación y la superación. Psicoperspectivas, 13(3), 93105. Recuperado de: https://dx.doi.org/10.5027/psicoperspectivas-Vol13-Issue3-fulltext-348 Celis, R. y Rodríguez, M. (2016). Constructivismo y construccionismo social en psicoterapia: Una perspectiva crítica. Bogotá, Colombia: Manual Moderno.

Domínguez, E., y Herrera, J. (2013). La investigación narrativa en psicología: definición y funciones.

Psicología desde el caribe 30(3), 620-641. Recuperado de : http://www.scielo.org.co/scielo.php?script=sci_arttext\&pid=S0123-417X2013000300009\&lng=en\&tIng=.

Estupiñán, J. y González, O. (2015). Narrativa conversacional, relatos de vida y tramas humanos. Bogotá, Colombia: Ediciones USTA.

Garzón, D. (2008). Autorreferencia y estilo terapéutico: su intersección en la formación de terapeutas sistémicos. Revista Diversitas, perspectivas en psicología, 4(1), 159- 171. Recuperado de http://www. scielo.org.co/pdf/dpp/v4n1/v4n1a14.pdf

Gaviria, A. (2015). Representaciones sociales de abuso sexual elaboradas por los niños, niñas, adolescentes y las familias que reportaron su caso en la Comisaría del Municipio de Isnos durante los años 2013 y 2014. (tesis de pregrado) Universidad Nacional Abierta y a Distancia UNAD, Colombia. Recuperado de: https://repository.unad.edu.co/bitstream/10596/3396/1/36291662.pdf

Instituto Colombiano de Bienestar Familiar. (2017). Lineamiento técnico del modelo para la atención de los niños, niñas y adolescentes, con derechos inobservados, amenazados, o vulnerados. Recuperado de https://www.icbf.gov.co/sites/default/files/procesos/document_19.pdf

Instituto Colombiano de Bienestar Familiar. (2018). Anexo del lineamiento técnico administrativo de ruta de actuaciones para el restablecimiento de derechos de niños, niñas y adolescentes víctimas de violencia sexual. Recuperado de https://www.icbf.gov.co/sites/default/files/anexo_ruta_violencia_sexual_17042018.pdf Instituto Nacional de Medicina Legal y Ciencias Forenses. (2019). Boletín Estadístico Mensual Di- 
ciembre 2019. Recuperado de https://www.medicinalegal.gov.co/documents/20143/349426/diciembre-2019.pdf/320ad04c-7c85-287f-804a-a49b1031d9f3

Kamsler, A. (1990). La formación de la imagen de sí misma. Terapia con mujeres que sufrieron abuso sexual durante la infancia. En M, Durrant y C, White (Ed.). Terapia del abuso sexual. (pp.15-53). Barcelona, España: Editorial Gedisa.

López, S. (2011). Terapias Breves: la propuesta de Michael White y David Epston. III Congreso Internacional de Investigación y Práctica Profesional en Psicología XVIII Jornadas de Investigación Séptimo Encuentro de Investigadores en Psicología del MERCOSUR. Facultad de Psicología - Universidad de Buenos Aires, Buenos Aires. Recuperado de https://www.aacademica.org/000-052/224 Pastor, M. y León, A.. (2007). Complejidad y Psicología social de las organizaciones. Psicothema, 19(2), 212-217. Recuperado de http://www.psicothema.com/pdf/3350.pdf

Payne, M. (2002). Terapia Narrativa: Una introducción para profesionales. Barcelona, España: PAIDÓS. Pereda, N. y Sicilia, L. (2017). Reacciones sociales ante la revelación de abuso sexual infantil y malestar psicológico en mujeres víctimas. Psychosocial Intervention, 26 (3), 131-138. Recuperado de http://www. redalyc.org/articulo.oa?id=179853469001

Puerto, M. (2015). Susurros de papel (Tesis de pregrado) Universidad de los Andes, Bogotá, Colombia. Recuperado de https://documentodegrado.uniandes.edu.co/documentos/7502.pdf

Quecedo, R. y Castaño, C. (2002). Introducción a la metodología de investigación cualitativa. Revista de Psicodidáctica, 14 (14), 5-39. Recuperado de http://www.redalyc.org/pdf/175/17501402.pdf Quiroz, M. y Peñaranda, F. (2009). Significados y respuestas de las madres al abuso sexual de sus hijas(os). Revista Latinoamericana de Ciencias Sociales, Niñez y Juventud, 7 (2), 1027-1053. Recuperado de http://www.redalyc.org/articulo.oa?id=77315614020

Rodríguez. L (2003). Intervención interdisciplinaria en casos de abuso sexual infantil. Universitas Psychologica, 2(1), 57-70. Recuperado de https://www.redalyc.org/articulo.oa?id=64720108 Rodríguez, C. (2015). Menores de 14 años víctimas de abuso sexual en Colombia (Tesis de maestría). Universidad Militar Nueva Granada, Bogotá. Recuperado de https://repository.unimilitar.edu.co/bitstream/10654/6676/1/RODRIGUEZHERRERACA RLOSESTEBAN2015.pdf

Rúa, R., Pérez, V. y González, R. (2018). El abuso sexual Infantil: opinión de los/as profesionales en contextos educativos. Prisma social revista de ciencias sociales, (23), 46-65. Recuperado de https://revistaprismasocial.es/article/view/2764

Sellado, M. (2015). A negociação da moralidade por meio da produção de justificativas na reconstrução da narrativa do abuso sexual de crianças e de adolescentes: um estudo situado. Revista Brasileira de Linguística Aplicada, 15 (3), 873-898. Recuperado de: http://www.redalyc.org/pdf/3398/339842232014. pdf

Sinclair, C. y Martínez, J. (2006). Culpa o Responsabilidad: Terapia con Madres de Niñas y Niños que han Sufrido Abuso Sexual. Psykhe, 15 (2), 25-35. Recuperado de http://www.redalyc.org/articulo. oa?id=96715203

Velázquez, V., Delgadillo, G. y González, V. (2013). Abuso Sexual Infantil, Técnicas básicas para su atención. Revista Reflexiones 92 (1), 131-139. Recuperado de:https://revistas.ucr.ac.cr/index.php/reflexiones/article/view/8831

Villanueva, I., Guzmán, P., Alonso, M., Beltrán, F. Gómez, G. y Pérez, I. (2011). Funcionamiento familiar en familias víctimas de abuso sexual intrafamiliar-incesto. Psicogente, 14 (25), 100-121. Recuperado de http://www.redalyc.org/articulo.oa?id=497552358010 